\title{
Thyroid Lymphosonography: A Novel Method for Evaluating Lymphatic Drainage
}

\author{
Joseph M. Curry, MD; Waleed H. Ezzat, MD; Daniel A. Merton, BS, RDMS; \\ Barry B. Goldberg, MD; David M. Cognetti, MD; David Rosen, MD; \\ Edmund A. Pribitkin, MD
}

Objectives: We evaluated lymphosonography, or contrast-enhanced, ultrasonography (US)-guided sentinel lymph node (SLN) detection, as a technique for demonstrating the lymphatic drainage of the thyroid gland.

Methods: In this prospective animal study, four 50-kg Yorkshire swine underwent transcutaneous injection of a US contrast agent and methylene blue dye into the thyroid gland. Contrast-enhanced US was used to identify draining lymphatic channels and SLNs. Sentinel node biopsy was conducted.Subsequently, bilateral neck and upper mediastinal dissection was carried out.

Results: In 3 of 4 cases, a blue dye-positive and US contrast-positive SLN was identified. We identified SLNs in level IV in 2 cases. One case revealed 2 adjacent nodes in the superior mediastinum. In 1 case, a lymphatic channel was identified traveling into the mediastinum, but exposure of the SLN could not be obtained. Noresidualblue dye-positive or US contrast-positive nodes were identified on subsequent dissection.

Conclusions: Lymphosonography of the thyroid gland in a porcine model correlates well with blue dye-guided sentinel node biopsy and is technically feasible, although in some cases access to the SLN may be difficult. This technique could potentially enable a detailed analysis of thyroidal lymphatic drainage if applied to humans.

Key Words: lymphosonography, sentinel node biopsy, thyroid.

\section{INTRODUCTION}

Traditional techniques of sentinel lymph node (SLN) biopsy primarily consist of blue dye injection and lymphoscintigraphy, and, though widely successful, these are not without technical challenges. Problems such as nonsequential enhancement (staining or uptake of second-echelon nodes), "shinethrough" (interference from a closely approximated injection site), ${ }^{2}$ and difficulty pinpointing subcentimeter nodes accurately have all been noted with application of this technique to the head and neck 3 Such difficulties can occur at other sites, as well, and thus have helped prompt a search for alternative methods of performing SLN biopsy.

Among these alternative techniques is lymphosonography, or contrast-enhanced, ultrasonography (US)-guided lymphatic mapping. The technique utilizes a microbubble contrast agent and conventional US techniques to identify SLNs draining cu- taneous, mucosal, or parenchymal lesions. Unlike other methods, this technique offers the unique ability to directly visualize both the lymphatic channels (LCs) and the lymph nodes by use of US. Goldberg et al 4,5 were the first to describe lymphosonography using a microbubble contrast agent (Sonazoid, GE Healthcare, Oslo, Norway) at various sites in animal models, and these early studies suggested a strong correlation with blue dye injection and lymphoscintigraphy. Moreover, on the basis of transcutaneous preoperative US appearance alone, metastatic tumor deposits were correctly predicted in 19 of 20 melanoma-positive nodes. 5 This technique could potentially allow for a highly sensitive and minimally invasive staging method for subclinical nodal disease. Nevertheless, such results remain investigatory, as the contrast agent is not yet available for human use in the United States, although phase 1 trials have shown it to be relatively safe for intravenous injection. 6

From the Department of Otolaryngology-Head and Neck Surgery, Thomas Jefferson University, Philadelphia, Pennsylvania. This study was performed in accordance with the PHS Policy on Humane Care and Use of Laboratory Animals, the NIH Guide for the Care and Use of Laboratory Animals, and the Animal Welfare Act (7 U.S.C. et seq.); the animal use protocol was approved by the Institutional Animal Care and Use Committee (IACUC) of Thomas Jefferson University.

Presented at the 7th International Conference on Head and Neck Cancer, Annual Meeting of the American Head and Neck Society, San Francisco, California, July 20, 2008.

Correspondence: Edmund A. Pribitkin, MD, Thomas Jefferson University, Dept of Otolaryngology-Head and Neck Surgery, 925 Chestnut St, 6th Floor, Philadelphia, PA 19107. 
Lymphatic metastasis from well-differentiated thyroid carcinoma is of little clinical significance in low-risk cases, $, 7,8$ despite its $30 \%$ to $90 \%$ prevalence (depending on disease stage and method of assessment). ${ }^{9}$ Although such regional metastasis may be associated with a greater rate of locoregional recurrence, it likely only has prognostic significance in those more than 45 years of age.${ }^{10}$ Typically, the ipsilateral central compartment nodes are involved, but some studies indicate that nearly $10 \%$ of patients may have "skip" metastases to the lateral neck. ${ }^{11}$

Several studies have investigated the use of SLN biopsy for well-differentiated thyroid carcinoma, ${ }^{12-14}$ but the technique has not demonstrated clinical utility or patient benefit. However, a technique such as lymphosonography may offer a valuable opportunity to precisely map lymphatic drainage patterns from the thyroid gland. Moreover, if easily obtained on preoperative US, pathologic confirmation of nodal status may be of some vahue in higher-risk cases. Additionally, this technique could possibly be of some benefit in intraoperative guid 2 ance during central compartment neck dissection. Furthermore, medullary thyroid carcinoma requires dissection of the cervical lymphatics, and SLN biopsy could, if clinically validated, have implications for the management of this disease. For these reasons, we sought to conduct a pilot study using blue dye and US contrast injections into the thyroid gland to evaluate lymphatic drainage patterns in a porcine model. utilized a porcine model for lymphatic surgery of the neck. ${ }^{15,16}$ The procedure was designed to identify the SLN(s) by use of lymphosonography and methylene blue dye, to evaluate the utility of $7 y m-$ phosonography for investigating lymphatid drainage of the thyroid gland.

Animals, Anesthesia, and Monitoring. Four Yorkshire swine, 5 to 6 months of age and weighing approximately $50 \mathrm{~kg}$, were utilized for the experiment in a humane and ethical fashion. The animals were fasted overnight, and pre-anesthesia sedation was administered with buprenorphine hydrochloride (Buprenex; Siam Pharmaceuticals Co Ltd, Bangkok, Thailand) $0.01 \mathrm{mg} / \mathrm{kg}$ and tiletamine hydrochloride-zolazepam hydrochloride (Telazol; Fort Dodge Animal Health, Fort Dodge, Iowa) $3 \mathrm{mg} / \mathrm{kg}$. Anesthesia was induced with intravenous propofol 6 $\mathrm{mg} / \mathrm{kg}$ and was maintained with $2 \%$ to $3.5 \%$ isoflurane (Abbott Laboratories, Abbott, Illinois) titrated for effect after endotracheal intubation. Ventilation was maintained at 10 to $15 \mathrm{~mL} / \mathrm{kg}$ per minute at 10 to 15 breaths per minute. Heart rate and pulse oximetry were monitored (Vet Ox 4700, Sensor Devices, Inc, Waukesha, Wisconsin). Lactated Ringer solution was administered at $10 \mathrm{~mL} / \mathrm{kg}$ per hour during the procedure. The animals were euthanized at the completion of the experiments.

Ultrasonography and Experimental Procedure. The swine were placed in the supine position on the operating table, the thyroid gland was visualized on preoperative US, and Sonazoid and methylene blue dye were injected. Sonazoid was reconstituted with 2 mL of sterile water (estimated $10^{9}$ microbubbles per milliliter), and $1 \mathrm{~mL}$ of suspension was injected under US/guidance. Subsequently, $1 \mathrm{~mL}$ of methylene blue dye was injected into the same site, without intervening removal of the needle. Conventional grayscale US using an Elegra scanner(Siemens Medical Systems, Issaquah, Washington) and a 7.5-MHz flat linear array transducer was used before the injection of contrast agent, with adjustment for imaging parameters such as system gain and depth of field. After contrast injection, grayscale pulse inversion harmonic imaging was used. This technique allows clear visualization of contrast agent flowing through the LCs with good accuracy and spatial resolution. For example, contrast agent can be clearly seen flowing into a nonenhancing lymph node. The mechanical index is a measure of the acoustic pressure generated Within the US field. ${ }^{17}$ The mechanical index values used for grayscale pulse-inversion harmonic inaging ranged from 0.2 to 0.5 , as microbubble rupture occurs at higher mechanical indices. Color flow Doppler US was performed with a higher mechanical index (less than 1.0), generating a heterogeneous Doppler signal as microbubbles are ruptured, thereby confirming the presence of contrast agent. All precontrast and postcontrast scans were performed by the same sonographer (D.A.M.).

Once contrast agent was identified at the injection site, the site was gently massaged to expedite flow of the contrast agent into the LCs and to the SLN(s). After identification of the SLN, both sides of the neck were examined with the probe to identify any additional contrast-positive nodes. Sentinel lymph node biopsy was performed with US guidance and visual identification of the blue dye through a small incision over the SLN (approximately $3 \mathrm{~cm}$ in length, with length based on the size of the lymph node). Once the SLNs were removed, an apron incision was made and both sides of the neck and the superior mediastinum were dissected. This allowed for thorough examination of levels I through IV bilaterally to identify any residual contrast agent- or blue dye-positive nodes. If the LC was followed into the 
Fig 1. Extended-field ultrasound view of injection site shows lymphatic channel (arrows) and sentinel lymph node filling with contrast agent (arrowhead).

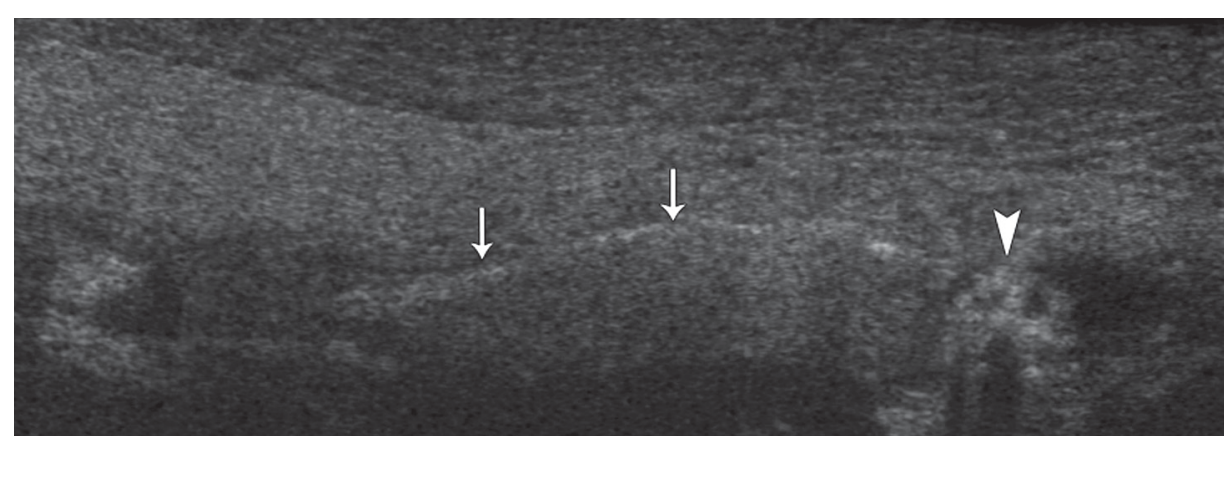

mediastinum and the SLN could not be reached via a small incision, no SLN biopsy was performed. Neck dissection and mediastinal exploration were subsequently initiated. The animal was then euthanized.

\section{RESULTS}

Four procedures were performed in 4 swine. After injection of the US contrast agent, transcutaneous US identified the injection site and that area was inspected until an LC was identified. Typically, flow was seen immediately after injection, but if necessary, the site was massaged gently to increase the rate of lymphatic flow of the contrast agent. The injection site appeared hyperechoic (ie, contrast-enhancing) after injection of the contrast agent, and a fine line of contrast agent flowing away from the site identified an LC on real-time contrast-enhanced US (Fig 1). As the contrast agent filled the SLN, the node became hyperechoic. Adjacent nonenhancing lymph nodes, blood vessels, and other critical structures could be identified, as could the depth of the node beneath the skin. This allowed noninvasive construction of a 3-dimensional map of the SLN's location before the biopsy. If an LC was identified, as occurs in most cases, flow to the SLN could be seen in real time. If no LCs were seen initially and the SLN was not identified, the entire neck could be systematically searched to find the SLNs. Contrast agent typically flowed directly to the SLN, and only rarely was any branching seen in the ICs.

The results of the procedures are summarized in the Table. In each case, flow into a single LC was identified by contrast-enhanced US in about a minute (mean, 1.25 minutes) after injection of the US contrast agent. In 2 of the 4 cases, a single level IV SLN was identified within 3 minutes on preoperative US (Figs 2 and 3 ). In the remaining 2 cases, the LC was followed inferiorly into the mediastinum, and this precluded transcutaneous contrast-enhanced US identification of an SLN. In 1 case, 2 SLNs containing both methylene blue dye and US contrast agent were identified on open neck dissection in the superior mediastinum. In the final case, an LC containing US contrast agent and methylene blue dye was identified traveling into the mediastinum, but flowed inferiorly outside the range of surgical dissection, and an SLN could not be harvested. Correlation between the lymphosonographic and blue dye-guided SLN biopsies occurred in 4 of 4 SLNs (Figs 2 and 3). No serious complications occurred during the proce-

RESULTS OF LYMPHOSONOGRAPHIC AND BLUE DYE-GUIDED SENTINEL NODE BIOPSY OF THYROID

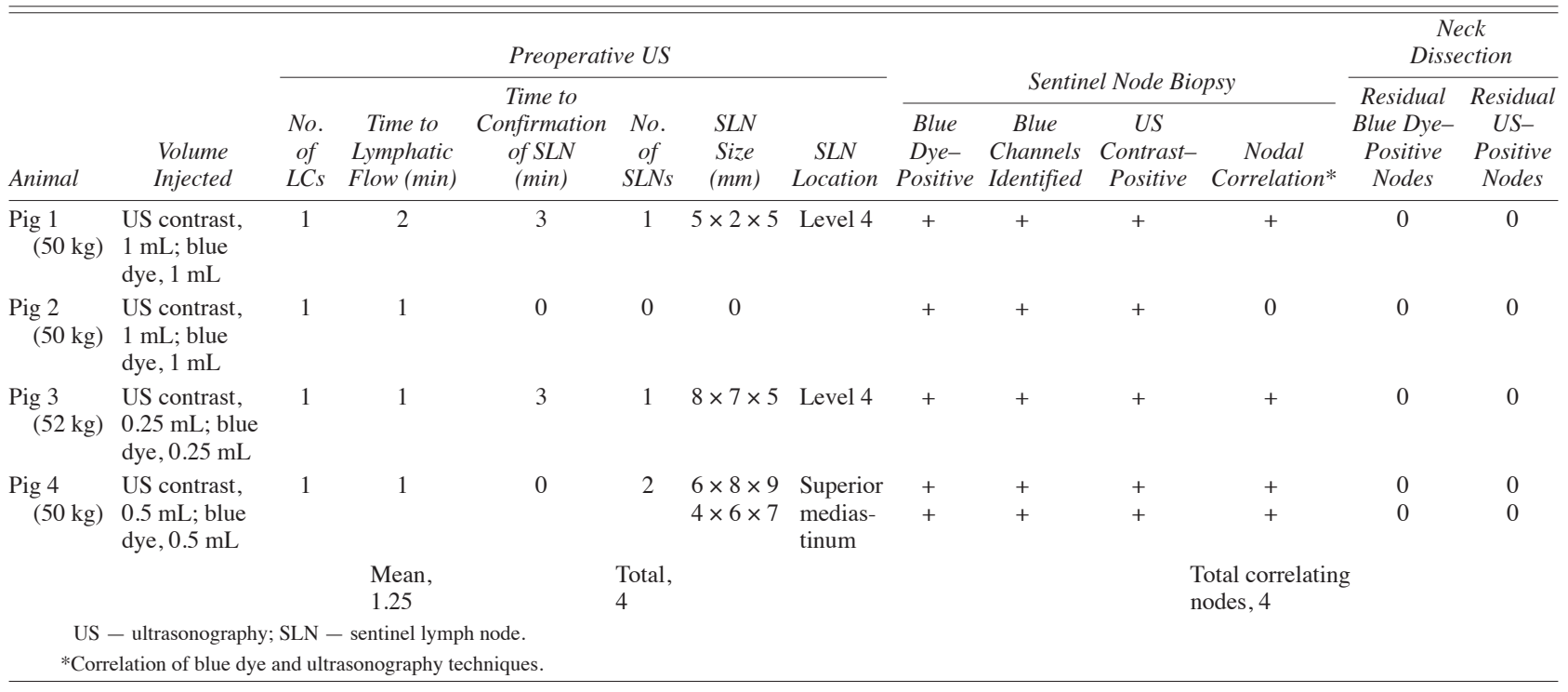




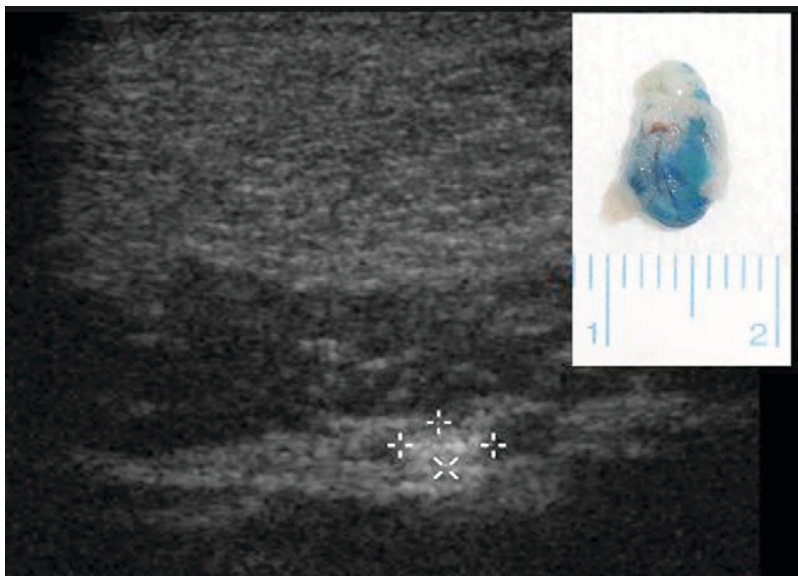

Fig 2. Sentinel lymph node is enhanced with ultrasound contrast agent on preoperative lymphosonography. Inset) Sentinel lymph node after surgical dissection.

dures, and no significant blood loss occurred. Upon open dissection of the neck, no residual US contrast-positive nodes or blue dye-stained nodes were identified. The residual nodal packet was closely examined, and all identified non-contrast-enhancing nodes were sectioned to identify blue dye within the nodes not visible on the external surface.

\section{DISCUSSION}

This pilot study describes successful LC mapping for the thyroid gland in a porcine model. Additionally, SLN biopsy could be successfully accomplished when the SLNs were identified in the neck and the upper mediastinum. All 4 US contrast-positive SLNs concentrated blue dye, and no blue-stained lymph nodes were identified that were not US contrast-positive. Although correlation of the SLN was not confirmed in the fourth procedure, this proce dure was limited by our ability to surgically extract

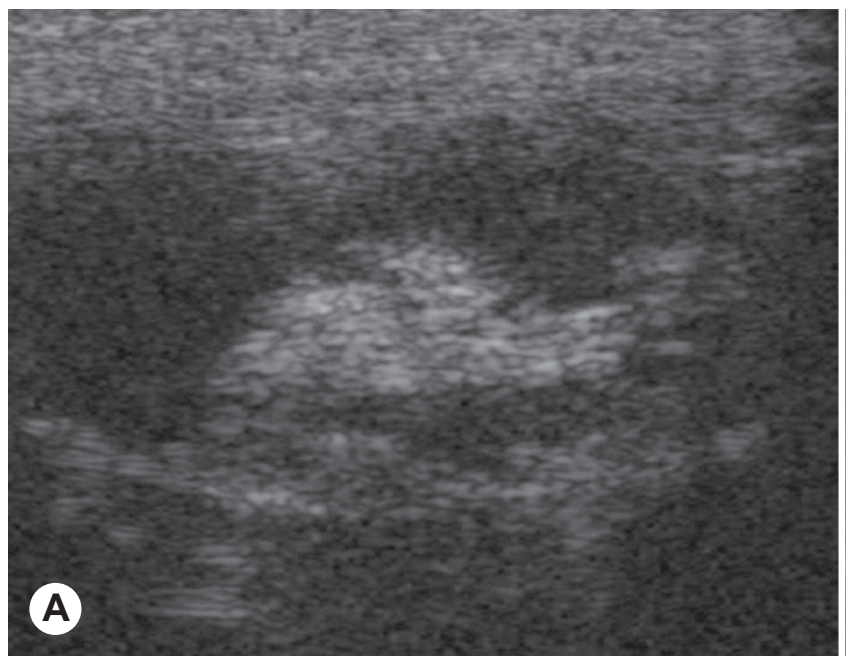

a node from the inferior mediastinum despite the identification of a blue dye-positive, US contrastpositive LC.

In addition to offering an opportunity to study undisturbed lymphatic drainage patterns in a highly detailed fashion, lymphosonography may have several relevant applications in the treatment of human well-differentiated thyroid carcinoma. Although the status of the regional lymph nodes in most patients is of questionable clinical significance, in higherrisk cases nodal metastasis may have some prognostic impact. ${ }^{18}$ Contrast-enhanced US could potentially allow for a detailed preoperative assessment of nodal status. The findings of Goldberg et $\mathrm{al}^{4}$ suggest that this technique may provide an accurate assessment of subclinical nodal disease in swine with melanoma, as they correctly predicted small foci of metastasis in 19 of 20 nodes based on US characterîstics alone. Contrast-enhanced US could conceivably permit preoperative detection of small metastatic foci within lymph nodes in cases of papillary carcinoma. Alternatiyely, US-directed fine-needle aspiration of the SLN could direct surgical intervention. Accordingly, this could limit the need for nodal dissection in appropriately selected patients, thereby reducing the risks associated with central compartment dissection, such as hypoparathyroidism or recurrent laryngeal nerve injury.

Additionally, there are a number of potential intraoperative applications. A lymphosonography-directed neck dissection could also be accomplished to ensure the harvest of SLNs during dissection. This could be particularly useful in the setting of aberrant lymphatic drainage patterns.

raphy that have been confirmed in previous stud-

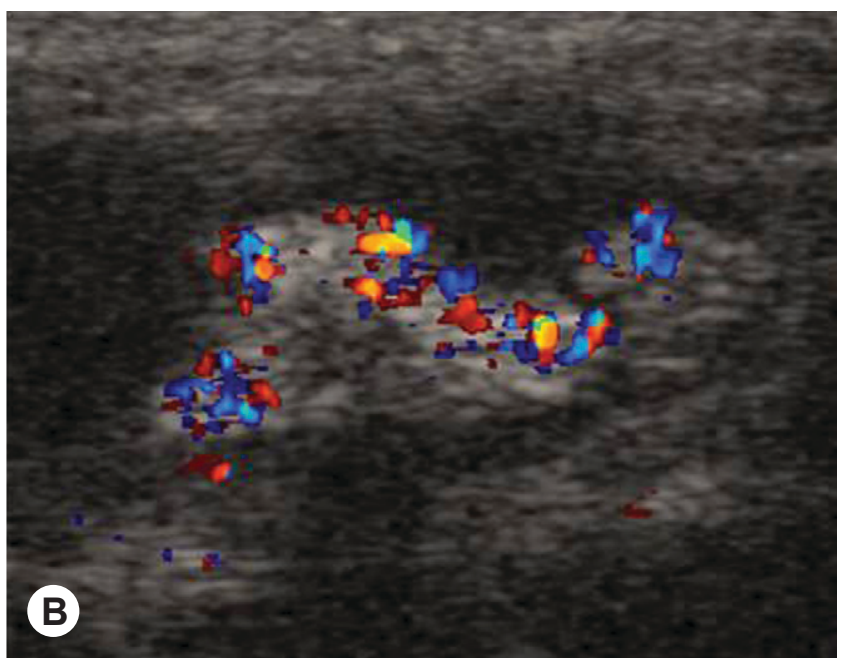

Fig 3. A) Grayscale ultrasound shows contrast agent within lymph node. B) Confirmation of ultrasound contrast agent using color flow Doppler demonstrates heterogeneous signal caused by microbubble rupture. 
ies were evident during the procedures performed in this experiment. ${ }^{4,5,15}$ The preprocedure US provided a highly detailed 3-dimensional map, allowing real-time visualization of contrast agent flowing into SLNs. Adjacent vascular structures were clearly visualized, as were nonenhancing lymph nodes and detailed architecture of the SLN. This technique proved exceptionally useful for nodal localization both before and during biopsy, enabling rapid dissection of the lymph node. Moreover, the "shine-through" effect frequently noted with the use of lymphoscintigraphy does not occur with lymphosonography. ${ }^{2}$ Additionally, no nonsequential nodal enhancement occurs with lymphosonography. As blue dye and radiocolloid are not trapped in the SLN, they can pass through to second-echelon nodes. This nonsequential enhancement can require harvest of additional lymph nodes, additional surgical dissection, and the risk of complications.

Furthermore, by allowing noninvasive visualization of in vivo LCs and architecture of the lymph nodes, this technique may increase our understanding of lymphatic drainage of the thyroidgland. For example, in this experiment, $1 \mathrm{LC}$ stained with blue dye and containing US contrast led into the inferior mediastinum and represented an atypical drainage pattern as compared to expected human patterns. It would be of clinical interest to determine the occurrence and frequency of atypical drainage patterns in humans.

Nevertheless, there are several limitations of this study. The primary limitation is the small number of procedures performed. A larger number of procedures may reveal clearer patterns of nodal drainage. A further limitation is the occurrence of the inferior mediastinal drainage pattern in 1 animal. Drainage to an SLN with correlation of blue dye and US contrast could not be confirmed in this case. The fact that the LCs contained both blue dye and US contrast suggests that correlation would have occurred, but no conchusions can be drawn. Perhaps a more suitable model for thyroid studies could be identified before investigation of the human thyroid gland is undertaken. The majority of lymphatic drainage is believed to occur in the ipsilateral central compartment, followed by the laterat neck and the superior mediastinum. A greater number of experiments may allow for more successful SENbiopsy without open exploration.

Lymphosonography of the thyroid gland in a porcine model is technically feasible, although limited by the porcine anatomy. This technique yields results comparable to those of blue dye-guided SLN biopsy. If clinically validated, this technique could offer a valuable technique for studying lymphatic drainage of the thyroid gland in great detail.

\section{REFERENCES}

1. Jansen L, Koops HS, Nieweg OE, et al. Sentinel node biopsy for melanoma in the head and neck region. Head Neck 2000;22:27-33.

2. Civantos F, Zitsch R, Bared A. Sentinel node biopsy in oral squamous cell carcinoma. J Surg Oncol 2007;96:330-6.

3. Fincher TR, Q'Brien JC, McCarty TM, et al. Patterns of drainage and recurrence following sentinel lymph node biopsy for cutaneous melanoma of the head and neck. Arch Otolaryngol Head Neck Surg 2004;130:844-8.

4. Goldberg BB, Merton DA, Liu JB, et al. Sentinellymph nodes in a swine model with melanoma: contrast-enhanced lymphatic US. Radiology 2004;230:727-34

5. Goldberg BB, Merton DA, Liu JB, Murphy G, Forsberg F. Contrast-enhanced sonographic imaging of lymphatic channels and sentinel lymph nodes. J Ultrasound Med 2005;24:95365.

6. Needleman L, Blomley MJ, Albrecht T, et al. Detection of liver lesion with ultrasound contrast agent Sonazoid: results of a multicenter study [Abstract]. J Ultrasound Med 2001;20:S11.

7. Shah JP, Loree TR, Dharker D, Strong EW, Begg C, Vlamis V. Prognostic factors in differentiated carcinoma of the thyroid gland. Am J Surg 1992;164:658-61.

8. Lundgren CI, Hall P, Dickman PW, Zedenius J. Clinically significant prognostic factors for differentiated thyroid carcinoma: a population-based, nested case-control study. Cancer 2006;106:524-31.
9. Caron NR, Tan YY, Ogilvie JB, et al. Selective modified radical neck dissection for papillary thyroid cancer - is level I, II and V dissection always necessary? World J Surg 2006;30: 833-40.

10. Hughes CJ, Shaha AR, Shah JP, Loree TR. Impact of lymph node metastasis in differentiated carcinoma of the thyroid: a matched-pair analysis. Head Neck 1996;18:127-32.

11. Roh JL, Kim JM, Park CI. Lateral cervical lymph node metastases from papillary thyroid carcinoma: pattern of nodal metastases and optimal strategy for neck dissection. Ann Surg Oncol 2008;15:1177-82.

12. Kelemen PR, Van Herle AJ, Giuliano AE. Sentinel lymphadenectomy in thyroid malignant neoplasms. Arch Surg 1998; 133:288-92.

13. Dixon E, McKinnon JG, Pasieka JL. Feasibility of sentinel lymph node biopsy and lymphatic mapping in nodular thyroid neoplasms. World J Surg 2000;24:1396-401.

14. Pelizzo MR, Boschin IM, Toniato A, et al. The sentinel node procedure with Patent Blue V dye in the surgical treatment of papillary thyroid carcinoma. Acta Otolaryngol 2001;121:4214.

15. Curry JM, Bloedon EA, Malloy KM, et al. Ultrasoundguided contrast-enhanced sentinel node biopsy of the head and neck in a porcine model. Otolaryngol Head Neck Surg 2007; 137:735-41.

16. Malloy KM, Cognetti DM, Wildemore BM, et al. Feasi- 
bility of endoscopic sentinel node biopsy in the porcine neck. Otolaryngol Head Neck Surg 2007;136:806-10.

17. McCulloch M, Gresser C, Moos S, et al. Ultrasound contrast physics: a series on contrast echocardiography, article 3 . J
Am Soc Echocardiogr 2000;13:959-67.

18. Shaha AR. Prognostic factors in papillary thyroid carcinoma and implications of large nodal metastasis. Surgery 2004; 135:237-9.

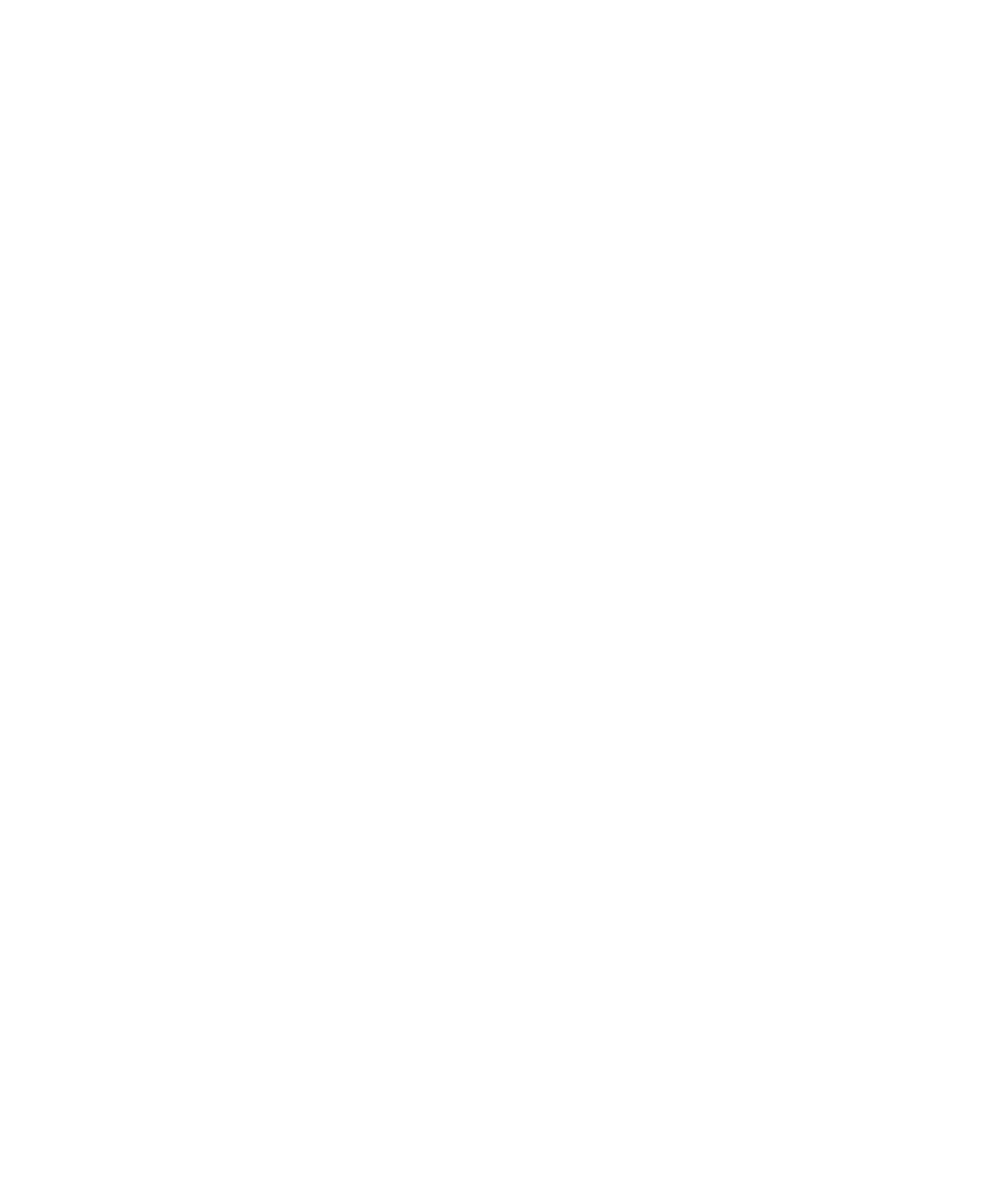

